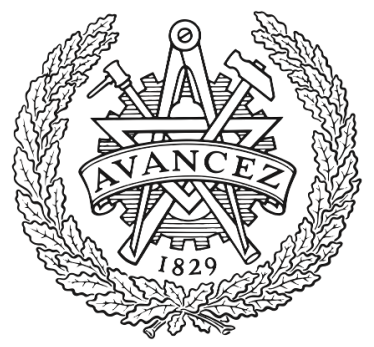

CHALMERS

UNIVERSITY OF TECHNOLOGY

\title{
Electron Beam Driven Generation of Frequency-Tunable Isolated Relativistic Subcycle Pulses
}

Downloaded from: https://research.chalmers.se, 2023-04-26 11:12 UTC

Citation for the original published paper (version of record):

Thiele, I., Siminos, E., Fülöp, T. (2019). Electron Beam Driven Generation of Frequency-Tunable Isolated Relativistic Subcycle Pulses. Physical Review Letters, 122(10).

http://dx.doi.org/10.1103/PhysRevLett.122.104803

N.B. When citing this work, cite the original published paper. 


\title{
Electron Beam Driven Generation of Frequency-Tunable Isolated Relativistic Subcycle Pulses
}

\author{
I. Thiele, ${ }^{1, *}$ E. Siminos, ${ }^{2, \dagger}$ and T. Fülöp ${ }^{1}$ \\ ${ }^{1}$ Department of Physics, Chalmers University of Technology, SE-412 96 Göteborg, Sweden \\ ${ }^{2}$ Department of Physics, University of Gothenburg, SE-412 96 Göteborg, Sweden
}

(Received 13 June 2018; revised manuscript received 30 August 2018; published 14 March 2019)

\begin{abstract}
We propose a novel scheme for frequency-tunable subcycle electromagnetic pulse generation. To this end a pump electron beam is injected into an electromagnetic seed pulse as the latter is reflected by a mirror. The electron beam is shown to be able to amplify the field of the seed pulse while upshifting its central frequency and reducing its number of cycles. We demonstrate the amplification by means of 1D and 2D particle-in-cell simulations. In order to explain and optimize the process, a model based on fluid theory is proposed. We estimate that using currently available electron beams and terahertz pulse sources, our scheme is able to produce millijoule-strong midinfrared subcycle pulses.
\end{abstract}

DOI: 10.1103/PhysRevLett.122.104803

The generation of few cycle electromagnetic pulses has steadily advanced, driven by applications which require probing or control of ultrafast processes [1,2]. Recently a lot of effort has been devoted to producing subcycle pulses in which the time envelope is modulated at timescale shorter than a single cycle. Such pulses bring temporal resolution to its ultimate limits and are unique tools for the control of electron motion in solids [3], electron tunneling in nanodevices [4], reaction dynamics at the electronic level [5], as well as the generation of isolated attosecond and zeptosecond x-ray pulses [6]. Several methods like optical synthesis or parametric amplification have been developed for the generation of subcycle pulses from the terahertz to $\mathrm{x}$-ray regimes (see the review [7]). While for few-cycle pulse durations these methods can lead to millijoule pulse energies, the energies of subcycle pulses are limited to a few microjoule. The main limitation of the typically used parametric amplification methods is the material damage threshold under intense fields [8]. On the other hand, methods exploiting plasmas or electron beams as a frequency conversion medium, such as high-harmonic generation from solid targets [9], Thomson scattering amplification [10], scattering by relativistic mirrors [11], and frequency down-conversion in a plasma wake $[12,13]$, are not subject to a damage threshold. However, these methods are not able to generate isolated subcycle pulses.

In this Letter, we propose a method to generate frequency-tunable isolated subcycle pulses reaching relativistic intensities. We particularly focus on the midinfrared regime [14]. Such pulses would lead to an ultrastrong light-matter coupling and might enable the switching of light-matter interaction within less than one cycle of light for the observation of new quantum mechanical nonadiabatic phenomena [15] or high-harmonic and isolated zeptosecond pulse generation with a significantly extended frequency cutoff $[3,6]$. As visualized in Fig. 1, our scheme involves the interaction of a seed electromagnetic pulse with a short duration pump electron beam at a thin foil. The thin foil acts as a mirror reflecting the seed pulse, while the electron beam enters in the middle of the pulse and leads to its amplification in a copropagating configuration. As will be shown below, a substantial part of the electron beam energy can be transferred to the electromagnetic pulse, more than doubling its energy. Currently available singlecycle terahertz sources reaching millijoule-pulse energies for central frequencies up to $\nu_{\mathrm{THz}}=4 \mathrm{THz}$ can be employed to produce suitable seed pulses $[16,17]$. To obtain subcycle pulses of comparable energy and with the central frequency in the midinfrared, $10-\mathrm{MeV} \mathrm{nC}$

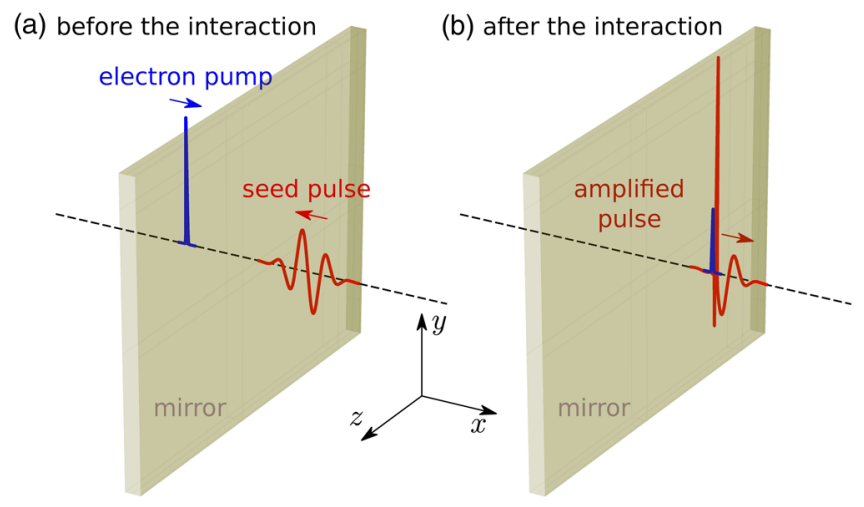

FIG. 1. Schematic representation of the electron beam driven amplification scheme. (a) The counterpropagating seed electromagnetic pulse and pump electron beam are moving towards a mirror (thin foil). (b) The electromagnetic pulse is reflected by the mirror and interacts with the electron beam as it exits through the mirror, leading to the generation and amplification of an intense subcycle pulse. 
electron bunches which are shorter than a single terahertz oscillation can be produced by compact laser-wakefield accelerators $[18,19]$.

We demonstrate the scheme through a 2D particle-incell (PIC) simulation with the code SMILEI [20]. A linearly polarized single-cycle seed pulse is focused strongly onto a thin almost perfectly reflecting foil. However, as will be clarified later on, our scheme can also operate with many-cycle seed pulses. The incoming seed pulse is focused at the mirror to obtain the $z$-polarized electric field $\mathbf{E}=E_{0}^{\text {in }} \exp \left(-y^{2} / y_{0}^{2}\right) \exp \left(-t^{2} / T_{0}^{2}\right) \sin \left(\omega_{0} t\right) \mathbf{e}_{z}$, with the field amplitude $E_{0}^{\text {in }}=E_{c}$, where $E_{c}=c m_{e} \omega_{0} / q_{e}, \omega_{0}=$ $2 \pi / T_{0}$ is the carrier frequency corresponding to the wavelength $\lambda_{0}=c T_{0}, y_{0}=0.3 \lambda_{0}$ characterizes the beam width, $t_{0}=0.21 T_{0}$ gives the time duration, and $\mathbf{e}_{z}$ is the unit vector along $z$. The electron beam is entering from the back side of the foil. It is initialized to a constant $\gamma$ factor $\gamma_{e}=20$ and a Gaussian density profile $n_{e}=$ $n_{e}^{\max } \exp \left(-y^{2} / y_{e}^{2}\right) \exp \left[-\left(x+\lambda_{0}\right)^{2} /\left(c t_{e}\right)^{2}\right]$ with thickness $y_{e}=0.3 \lambda_{0}$, duration $t_{e}=0.016 T_{0}$, and peak density $n_{e}^{\max }=28.3 n_{c}$, where $n_{c}=m_{e} \epsilon_{0} \omega_{0}^{2} / q_{e}^{2}$ is the critical density for a resting plasma. A snapshot of the electric field after the amplification process has been completed is presented in Fig. 2(a). We observe a strong subcycle pulse around $x=1.91 \lambda_{0}$. It is well collimated compared to the residual driving electromagnetic pulse which diffracts strongly due to the tight focusing. This is an advantageous property of the scheme because of the natural separation between the seed and amplified electromagnetic pulse. As the on-axis electric field time trace in Fig. 2(b) demonstrates, already after propagation for two seed wavelengths, the pulses are almost separated. The corresponding frequency spectrum in Fig. 2(c) shows that the generated subcycle pulse is upshifted by a factor of 7 in terms of peak frequency and is therefore diffracting much less than the seed pulse. The energy of the pulse is amplified by a factor of 2.4.

The process remains effective when using an electron beam with $150 \%$ energy spread, i.e., with a Maxwellianlike spectrum similar to those produced by laser-wakefield accelerators operating in the self-modulated regime $[18,19]$; see Figs. 2(b) and 2(c). For cold electron beams the overall efficiency $\eta=\left(\mathcal{E}_{\text {out }}-\mathcal{E}_{\text {seed }}\right) / \mathcal{E}_{e}$, where $\mathcal{E}_{\text {out }}$, $\mathcal{E}_{\text {seed }}$, and $\mathcal{E}_{e}$ are the outgoing, seed pulse, and electron beam energies, ranges from $1 \%$ for weak seed pulses up to $18 \%$ for stronger seed pulses [see Fig. 2(d)]. For Maxwellian-like electron beam spectra, the conversion efficiency is slightly lower, yet remains above $9 \%$ for strong seed pulses [see Fig. 2(d)], implying that such electron beams are still usable for the production of millijoule-level mid-IR subcycle pulses. Moreover, amplification is robust with respect to jitter effects [see Fig. 2(e)]. We note that the radiation reported here is distinct from transition radiation [22,23], which can dominate for weak seed pulses but has very different properties (see Supplemental Material [24]).
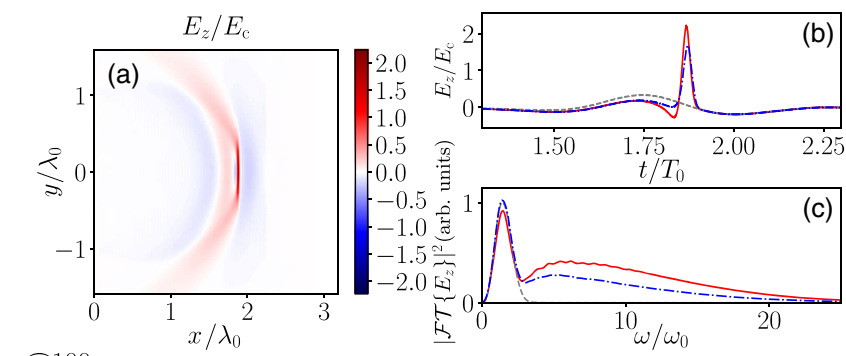

(b)

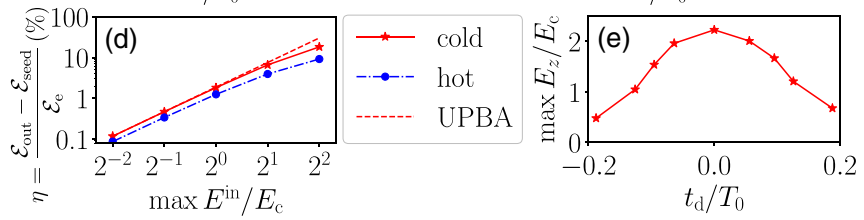

FIG. 2. (a) Electric field snapshot after the interaction of a strongly focused low-frequency pulse with a monoenergetic electron beam passing the standing mirror at $x=0$. Corresponding on-axis signal (b) and spectrum (c) demonstrating the generation of an intense higher-frequency subcycle pulse with a cold monoenergetic electron beam (solid red lines), a beam with $150 \%$ energy spread having the same total energy (dash-dotted blue lines), and without the electron beam (dashed gray lines). (d) Conversion efficiency vs seed field strength. (e) Amplified peak electric field dependence on delay time $t_{d}$. The parameters are $t_{e}=0.016 T_{0}, y_{e}=0.3 \lambda_{0}, n_{e}^{\max }=28.3 n_{c}, \gamma_{e}=20$, $t_{0}=0.21 T_{0}$, and $E_{0}^{\text {in }}=E_{c}$ [except in (d)]. The simulation was performed with 1600 points per electromagnetic pulse carrier wavelength along $x, 100$ points along $y, 1608$ points per electromagnetic pulse carrier oscillation, and 100 macroparticles per cell. We consider an aluminium foil of thickness $1.4 c / \omega_{0}$ acting as a mirror. Since such a foil does not stop mega-electronvolt electrons (Ref. [21], p. 376) and ensures the reflection of the low-frequency seed pulse, we simply model it as a dense electron plasma.

In order to illustrate why a standing mirror is required in addition to the electron beam in order to produce subcycle pulses, we consider the simplified space-time diagrams in Fig. 3. We restrict our attention to 1D geometry and consider the limit of an infinitely dense and sharply rising electron beam front. Without the standing mirror [Fig. 3(a)] the setup is known as the relativistic flying mirror concept [11]. The solid red line indicates the edges of the incoming electromagnetic pulse which is perfectly reflected by the electron beam. Because of the double Doppler shift effect, the frequency of the reflected pulse is upshifted and its amplitude amplified by a factor $\approx 4 \gamma_{e}^{2}$ [29]. While the duration of the reflected pulse is shortened by the same factor, the number of cycles remains invariant. By contrast, when the standing mirror is introduced [solid black line at $x=x_{0}$ in Fig. 3(b)], the leading part of the electromagnetic pulse is simply reflected and not amplified. Only the trailing part interacts with the electron beam and, thus, the number of amplified cycles is reduced.

We now present a simplified 1D $\left(\partial_{z}=\partial_{y}=0\right)$ fluid model of the interaction (for details see the Supplemental 


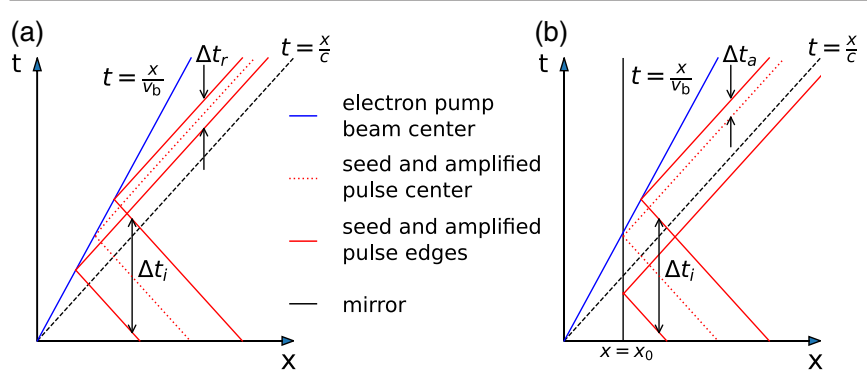

FIG. 3. Space-time diagrams visualizing the electron beam (blue line) moving with speed $v_{b}$ and electromagnetic pulse (red lines) without (a) or with (b) the standing mirror at $x=x_{0}$ [black line in (b)]: Without the mirror the whole electromagnetic pulse and with the mirror only part of the electromagnetic pulse interacts with the electron beam allowing for subcycle pulse generation.

Material [24]) in order to illuminate the mechanism of the electron beam driven amplification. The transverse fluid momentum $\mathbf{p}_{\perp}$ evolves according to

$$
\partial_{t} \mathbf{p}_{\perp}=q_{e} \mathbf{E}_{\perp},
$$

where $\mathbf{E}_{\perp}$ is the transverse electric field and $q_{e}$ is the electron charge. This leads to conservation of transverse canonical momentum, $\mathbf{p}_{\perp}=-q_{e} \mathbf{A}_{\perp}$, where $\mathbf{E}_{\perp}=-\partial_{t} \mathbf{A}_{\perp}$ and $\mathbf{A}_{\perp}$ is the vector potential (in the Coulomb gauge). The transverse current reads

$$
\mathbf{J}_{\perp}=\left(q_{e} / m_{e}\right) \mathbf{p}_{\perp} n_{e} / \gamma_{e},
$$

where $\gamma_{e}=\sqrt{1+|\mathbf{p}|^{2} /\left(m_{e} c\right)^{2}}, m_{e}$ is the electron mass and $c$ the speed of light in vacuum. If we choose a sufficiently weak seed pulse, the longitudinal momentum of the electrons dominates and $\gamma_{e} \approx p_{x} /\left(m_{e} c\right)$. This allows us to neglect the effect of the seed pulse on the longitudinal electron beam momentum, i.e., to employ an undepleted pump beam approximation (UPBA). Equation (1) is then solved together with Maxwell's equations with a source term given by Eq. (2) for a given electron beam dynamics with prescribed $n_{e}(x, t)$ and $v_{x}(x, t)=p_{x}(x, t) /$ $\left[m_{e} \gamma_{e}(x, t)\right]$. For simplicity, we assume a density profile moving with a constant speed $v_{b}$ and $\gamma_{e}$,

$$
n_{e}(x, t)=n_{e}^{\max } \exp \left[-\left(t-t_{d}-x / v_{b}\right)^{2} / t_{e}^{2}\right]
$$

with some delay $t_{d}$. The seed pulse arriving from $x=+\infty$ is perfectly reflected by the mirror at $x=0$; i.e., the electric field at the mirror is zero. The fields can be decomposed into forward and backward propagating parts $E_{z}^{-}$and $E_{z}^{+}$, respectively, such that the seed pulse electric field at the mirror is defined by

$$
E_{z}^{ \pm}(t)=\mp E_{z}^{\max } \sin \left(\omega_{0} t\right) \exp \left(-t^{2} / t_{0}^{2}\right)
$$
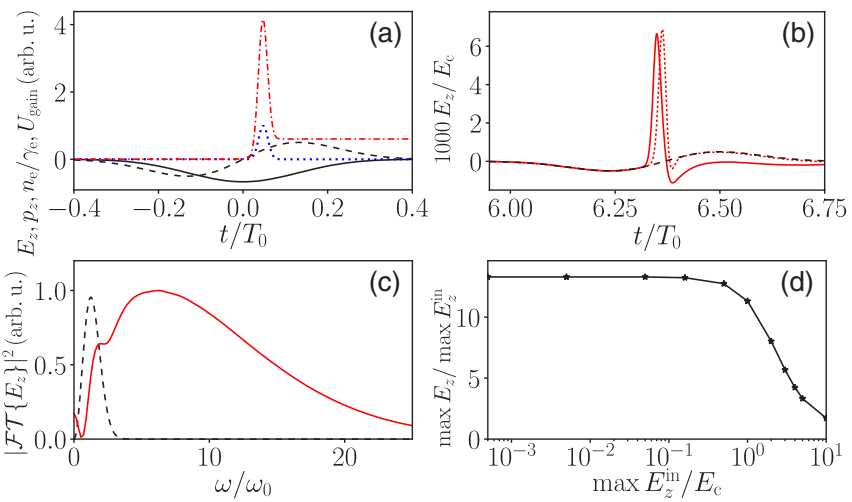

FIG. 4. (a) Visualization of the amplification mechanism. Electric field approximated by the unperturbed reflected seed pulse field (dashed line), corresponding vector potential (solid line), electron density (dotted line), and time-dependent energy density gain $U_{\text {gain }}$ (dash-dotted line). (b) Electric field after the interaction of the incoming plane wave single-cycle pulse (dashed line) with the electron beam according to the fluid model (dotted line) and PIC (solid line). (c) Spectra according to PIC simulation. The parameters are $t_{e}=0.016 T_{0}, t_{d}=0, n_{e}^{\max }=28.3 n_{c}$, $\gamma_{e}=20, t_{0}=0.21 T_{0}, E_{0}^{\text {in }}=0.001 E_{c}$. The distance from the mirror to the detector is $6.37 c / \omega_{0}$. (d) Peak electric field amplitude amplification for different incoming seed pulse amplitudes according to PIC simulations. The remaining parameters are the same as above.

In Fig. 4(a), an example of an $n_{e} / \gamma_{e}$ profile (dotted line) and an outgoing seed electric field (dashed line) is shown.

The solution of the fluid model for a weak seed pulse and a density profile shorter than the cycle duration is shown in Fig. 4(b). We indeed observe a partial amplification of the incoming seed pulse (dashed line), leading to the formation of a subcycle pulse in the center of the original pulse. After the interaction, the pulse energy increases by a factor of 10 and the maximum electric field of the electromagnetic pulse is enhanced by about a factor of 14 (dotted line). We compare the result of the fluid model with PIC simulations [dark red solid line in Figs. 4(b) and 2(d)] to find an excellent agreement which justifies the use of the fluid picture and the UPBA. As Fig. 4(c) shows, the spectrum of the reflected pulse is upshifted by a factor of 7 and strongly broadened.

From Poynting's theorem and Eqs. (1) and (2) we can compute the energy density $U_{\text {gain }}$ transferred to the electromagnetic field at any given point in space during the interaction as [24]

$$
U_{\text {gain }}(t)=\frac{q_{e}^{2}}{m_{e}} \int_{-\infty}^{t}\left|\mathbf{A}_{\perp}\right|^{2} \frac{\partial}{\partial \tau}\left(\frac{n_{e}}{\gamma_{e}}\right) d \tau
$$

Here we ignored terms that identically vanish after the end of the interaction. It is important to note that the sign of $U_{\text {gain }}$ at any given time only depends on the rate of change of $n_{e} / \gamma_{e}$. For a constant $\gamma_{e}$, the rising part of the electron beam gives a gain, while the descending part of the electron 
beam gives a loss. Assuming a symmetric electron beam profile, a net gain after the end of the interaction $(t \rightarrow \infty)$ requires an asymmetry in the amplitude of electromagnetic field vector potential $\left|\mathbf{A}_{\perp}\right|$. For a quantitative assessment $\mathbf{A}_{\perp}$ has to be determined by solving the full problem.

A great advantage of our scheme (see Fig. 1) is that the introduction of the standing mirror allows the electron beam to be injected into the seed pulse in a way that such an asymmetry, and thus a net gain, can be achieved. This is illustrated in Fig. 4(a) where, in order to explain the interaction in simple terms, we assumed the electric field $E_{z}$ to be the one of the unperturbed reflected seed pulse (dashed line). The corresponding transverse vector potential $\mathbf{A}_{\perp}=-\mathbf{p}_{\perp} / q_{e}$ for our example can then be directly computed from Eq. (1) and is also presented in Fig. 4(a) (solid line). This gives the evolution of $U_{\text {gain }}$ (dash-dotted line), which first increases at the rising edge of $n_{e} / \gamma_{e}$ [dotted line in Fig. 4(a)] and then decreases at the descending edge. Since in our example we have chosen a slightly positive electron beam delay $t_{d}=0.05 T_{0}$, a final local nonzero energy gain can be expected. We note that injecting the beam with $t_{d}=0$ as for Figs. 4(b) and 4(c) leads also to an energy gain after some propagation since the electron beam is slower than the seed pulse. In order to take into account other effects that may become important, such as the modification of the electric field due to its amplification or the contribution of the incoming part of the seed pulse, the full model needs to be solved.

For electron beam duration that is much shorter than the laser cycle we may Taylor expand $\mathbf{A}_{\perp}(x, t)$ in Eq. (5) around $t=t_{d}$ :

$$
U_{\text {gain }}(+\infty)=-\frac{q_{e}^{2}}{m_{e}} \frac{\partial}{\partial t}\left|\mathbf{A}_{\perp}\left(t_{d}\right)\right|^{2} \int_{-\infty}^{+\infty} n_{e} / \gamma_{e} d t
$$

This shows explicitly the dependence of the energy gain on the electromagnetic field profile when the bunch exits through the mirror. We see that, in this limit, the maximum energy gain is independent of the electron beam duration for a constant charge. Equation (5) can be used to predict many other trends, such as a decrease of the amplification for longer electron beams, the possibility to maintain the amplification using many-cycle seed pulses if $t_{d} \neq 0$, or using a chirp (see Ref. [24] for details).

The fluid model, within the UPBA, predicts a linear increase of the amplification with the amplitude of the seed pulse. As Figs. 2(d) and 4(d) show, this is true up to relativistic seed pulse amplitudes. This feature of our scheme implies the possibility to up-scale the amplitudes of the subcycle pulses up to relativistic intensities.

As the example in Fig. 5 demonstrates, our subcycle-pulse generation and amplification scheme works not only for single-cycle but also for few-cycle driving electromagnetic pulses. Actually, the scheme would lead to amplification for any duration of the driving pulse. As long as the electron
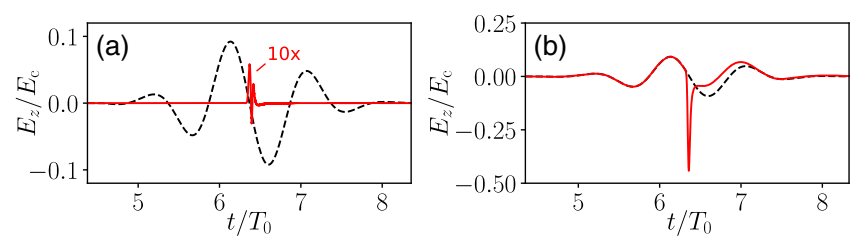

FIG. 5. Electric field time traces before (dashed lines) and after (solid lines) the interaction using a few-cycle seed pulse and an underdense electron beam without (a) or with (b) the mirror. The field after the interaction without the mirror [solid line in (a)] has been magnified by a factor of 10 for a better comparison. The parameters are $t_{e}=0.016 T_{0}, n_{e}^{\max }=5.66 n_{c}, \gamma_{e}=10$, $t_{0}=0.85 T_{0}, E_{0}^{\text {in }}=0.1 E_{c}$. The simulations were performed with 1600 points per electromagnetic pulse carrier wavelength and with 1000 particles per cell. The mirror has been modeled as a dense electron plasma with thickness $0.22 \lambda_{0}$.

beam duration is small compared to electromagnetic pulse cycle duration $2 \pi / \omega_{0}$, Eq. (6) predicts a potential energy gain independent of the number of cycles in $\mathbf{A}_{\perp}$.

The cases demonstrated in Figs. 4 and 5 also differ in terms of the maximum value for $n_{e} / \gamma_{e}$. In the former case, the electron beam is overdense $\left(n_{e}^{\max } / \gamma_{e}>n_{c}\right)$ for the carrier frequency $\omega_{0}$, while in the latter case it is underdense $\left(n_{e}^{\max } / \gamma_{e}<n_{c}\right)$; i.e., the reflectivity due to the electron beam itself is almost zero. Nevertheless, due to the mirror the incident electromagnetic pulse is fully reflected. Thus, its amplitude is amplified by a factor of 4.8 and the energy is doubled even in the underdense case. By contrast, without the mirror, the reflected pulse contains only $1.7 \%$ of the initial electromagnetic pulse energy, its amplitude is diminished by 20 times compared to the seed pulse, and no subcycle pulse is generated.

Up to now we presented our results in normalized units: in particular, frequencies were normalized to $\omega_{0}$, durations to $T_{0}=2 \pi / \omega_{0}$, electric fields to $E_{c}=c m_{e} \omega_{0} / q_{e}$, and densities to the critical density $n_{c}$. This implies the possibility to tune the frequency spectrum of the generated subcycle pulse with the input parameters. The output frequencies can be tuned proportionally with the seed carrier frequency $\omega_{0}$ if $n_{e} / \gamma_{e}$ is increased with $\omega_{0}^{2}$, the electron beam duration and transverse size are decreased with $1 / \omega_{0}$. This corresponds to a reduction of the beam charge and subcycle pulse energy with $1 / \omega_{0}$, but a peak electric field amplitude rise with $\omega_{0}$.

For the cases we are looking at in this Letter, the central frequency upshifts by about a factor of 10 . This leads to the frequency conversion key as presented in Table I, including the necessary bunch duration, transverse size, and charge computed from the electron density while assuming equal size in both transverse dimensions.

In summary, we have proposed a scheme for the generation of isolated, intense, subcycle pulses which is based on the interaction of an electron beam with a seed electromagnetic pulse reflected by a mirror. The mirror is a crucial element which allows us to introduce the electron 
TABLE I. Frequency conversion key between seed and amplified electromagnetic pulse for the subcycle generation mechanism.

\begin{tabular}{lcccccc}
\hline \hline Seed carrier & Frequency & Charge $(\mathrm{pC})$ & Transverse size $(\mu \mathrm{m})$ & Bunch duration $(\mathrm{fs})$ & Output & Central wavelength \\
\hline Terahertz & $1-10 \mathrm{THz}$ & $500-5000$ & $30-300$ & $10-100$ & (Mid)infrared & $3-30 \mu \mathrm{m}$ \\
(Mid)infrared & $10-100 \mathrm{THz}$ & $50-500$ & $3-30$ & $1-10$ & Optical & $300 \mathrm{~nm}-3 \mu \mathrm{m}$ \\
Optical & $100-1000 \mathrm{THz}$ & $5-50$ & $0.3-3$ & $0.1-1$ & EUV & $30-300 \mathrm{~nm}$ \\
\hline \hline
\end{tabular}

beam with the correct phase into the fully reflected seed pulse. This ensures an efficient energy conversion from the beam to the pulse leading up to relativistic intensities and down to subcycle duration. In particular, we have shown that using currently available intense terahertz pulse sources and laser-wakefield-accelerated electron beams, millijoule-strong midinfrared subcycle pulses can be generated. We believe that our proposed scheme will trigger further theoretical and experimental investigations of both intense subcycle pulse sources and applications.

The authors thank M. Grech for helpful discussions and the anonymous referees for helpful comments. This work was supported by the Knut and Alice Wallenberg Foundation, the European Research Council (ERC-2014-CoG Grant No. 647121), and by the Swedish Research Council, Grant No. 2016-05012. Numerical simulations were performed using computing resources at Grand Équipement National pour le Calcul Intensif (GENCI, Grants No. A0030506129 and No. A0040507594) and Chalmers Centre for Computational Science and Engineering (C3SE) provided by the Swedish National Infrastructure for Computing (SNIC, Grants No. SNIC 2017/1-484, No. SNIC 2017/1-393, and No. SNIC 2018/1-43).

*illia-thiele@web.de †evangelos.siminos@physics.gu.se

[1] P. B. Corkum and F. Krausz, Nat. Phys. 3, 381 (2007).

[2] F. Krausz and M. I. Stockman, Nat. Photonics 8, 205 (2014).

[3] M. Hohenleutner, F. Langer, O. Schubert, M. Knorr, U. Huttner, S. W. Koch, M. Kira, and R. Huber, Nature (London) 523, 572 (2015).

[4] T. Rybka, M. Ludwig, M. F. Schmalz, V. Knittel, D. Brida, and A. Leitenstorfer, Nat. Photonics 10, 667 (2016).

[5] M. F. Kling, C. Siedschlag, A. J. Verhoef, J. I. Khan, M. Schultze, T. Uphues, Y. Ni, M. Uiberacker, M. Drescher, F. Krausz, and M. J. J. Vrakking, Science 312, 246 (2006).

[6] C. Hernández-García, J. A. Pérez-Hernández, T. Popmintchev, M. M. Murnane, H. C. Kapteyn, A. Jaron-Becker, A. Becker, and L. Plaja, Phys. Rev. Lett. 111, 033002 (2013).

[7] C. Manzoni, O. D. Mucke, G. Cirmi, S. Fang, J. Moses, S.-W. Huang, K.-H. Hong, G. Cerullo, and F. X. Kartner, Laser Photonics Rev. 9, 129 (2015).

[8] D. E. Rivas et al., Sci. Rep. 7, 5224 (2017).

[9] U. Teubner and P. Gibbon, Rev. Mod. Phys. 81, 445 (2009).

[10] E. Esarey, S. K. Ride, and P. Sprangle, Phys. Rev. E 48, 3003 (1993).

[11] S. V. Bulanov, T. Z. Esirkepov, M. Kando, and J. Koga, Plasma Sources Sci. Technol. 25, 053001 (2016).
[12] F. S. Tsung, C. Ren, L. O. Silva, W. B. Mori, and T. Katsouleas, Proc. Natl. Acad. Sci. U.S.A. 99, 29 (2002).

[13] Z. Nie, C.-H. Pai, J. Hua, C. Zhang, Y. Wu, Y. Wan, F. Li, J. Zhang, Z. Cheng, Q. Su, S. Liu, Y. Ma, X. Ning, Y. He, W. Lu, H.-H. Chu, J. Wang, W. B. Mori, and C. Joshi, Nat. Photonics 12, 489 (2018).

[14] H. Liang, P. Krogen, Z. Wang, H. Park, T. Kroh, K. Zawilski, P. Schunemann, J. Moses, L. F. DiMauro, F. X. Kärtner, and K.-H. Hong, Nat. Commun. 8, 141 (2017).

[15] G. Günter, A. A. Anappara, J. Hees, A. Sell, G. Biasiol, L. Sorba, S. De Liberato, C. Ciuti, A. Tredicucci, A. Leitenstorfer, and R. Huber, Nature (London) 458, 178 (2009).

[16] C. Vicario, B. Monoszlai, and C. P. Hauri, Phys. Rev. Lett. 112, 213901 (2014).

[17] G. Liao, H. Liu, Y. Li, G. G. Scott, D. Neely, Y. Zhang, B. Zhu, Z. Zhang, C. Armstrong, E. Zemaityte, P. Bradford, P. G. Huggard, P. McKenna, C. M. Brenner, N. C. Woolsey, W. Wang, Z. Sheng, and J. Zhang, arXiv:1805.04369.

[18] A. J. Goers, G. A. Hine, L. Feder, B. Miao, F. Salehi, J. K. Wahlstrand, and H. M. Milchberg, Phys. Rev. Lett. 115, 194802 (2015).

[19] F. Salehi, A. J. Goers, G. A. Hine, L. Feder, D. Kuk, B. Miao, D. Woodbury, K. Y. Kim, and H. M. Milchberg, Opt. Lett. 42, 215 (2017).

[20] J. Derouillat, A. Beck, F. Prez, T. Vinci, M. Chiaramello, A. Grassi, M. Fl, G. Bouchard, I. Plotnikov, N. Aunai, J. Dargent, C. Riconda, and M. Grech, Comput. Phys. Commun. 222, 351 (2018).

[21] W. Heitler, The Quantum Theory of Radiation, 3rd ed. (Dover Publications, New York, 1954).

[22] J. D. Jackson, Classical Electrodynamics, 4th ed. (Wiley, New York, 2006).

[23] C. B. Schroeder, E. Esarey, J. van Tilborg, and W.P. Leemans, Phys. Rev. E 69, 016501 (2004).

[24] See Supplemental Material athttp://link.aps.org/supplemental/ 10.1103/PhysRevLett.122.104803 for more details on the simplified 1D model as well as some comments on transition radiation, which includes Refs. [22,23,25-28].

[25] A. Taflove, Computational Electrodynamics: The FiniteDifference Time-Domain Method, Antennas and Propagation Library (Artech House, Inc., Boston, 1995).

[26] W. P. Leemans, J. van Tilborg, J. Faure, C. G. R. Geddes, C. Tóth, C. B. Schroeder, E. Esarey, G. Fubiani, and G. Dugan, Phys. Plasmas 11, 2899 (2004).

[27] Z. Wu, A. S. Fisher, J. Goodfellow, M. Fuchs, D. Daranciang, M. Hogan, H. Loos, and A. Lindenberg, Rev. Sci. Instrum. 84, 022701 (2013).

[28] G.-Q. Liao, Y.-T. Li, Y.-H. Zhang, H. Liu, X.-L. Ge, S. Yang, W.-Q. Wei, X.-H. Yuan, Y.-Q. Deng, B.-J. Zhu, Z. Zhang, W.-M. Wang,Z.-M. Sheng,L.-M.Chen, X.Lu, J.-L.Ma, X. Wang, and J. Zhang, Phys. Rev. Lett. 116, 205003 (2016).

[29] K. Landecker, Phys. Rev. 86, 852 (1952). 